\title{
Investigation of the influence of the number of vanes on the performance of a rotary vane compressor
}

\author{
Andrey Kotlov ${ }^{1,}$, Leonid Kuznetsov ${ }^{2}$ and Boris Hrustalev 1 \\ ${ }^{1}$ Peter the Great St. Petersburg Polytechnic University, Polytechnicheskaya 29, St. Petersburg, \\ 195251, Russian Federation \\ ${ }_{2}^{2} J S C$ Scientific Production Association "Compressor", Bol'shoy Sampsoniyevskiy Prospekt, 64, St. \\ Petersburg, 194044, Russian Federation
}

\begin{abstract}
We developed a mathematical model for determining the parameters of mass transfer in the compressor chambers during the processes of compression and discharge. The mass flow rates through the end and radial gaps were determined. Also we analyzed the processes of mass transfer in a clamped volume. We investigated the influence of the number of vanes on the compressor efficiency, taking into account changes in the compressor geometric parameters. We established that overflows through the end surfaces of rotor mainly affect the compressor performance. In order to reduce overflows during the period of discharge, it was proposed to increase the angle of closure of the discharge window at a fixed angle of its opening. The mathematical model allows one to make recommendations on the choice of the optimal number of vanes for a particular design.
\end{abstract}

\section{Introduction}

Rotary vane compressors (RVC) belong to the class of volumetric machines. Interest in the production of RVC is due to the simplicity of its design, low cost of the manufacturing process, ease of operation and relatively high reliability $[1,2]$. At the same time, there is a challenge to increase the low efficiency of the RVC [3-7]. RVC are used in the metallurgical, chemical and gas industries, for pneumatic transport of bulk materials, in the food industry and trade, in brake systems of vehicles. They are also used as vacuum pumps in bulb and glass production, etc. They are widely used in refrigeration technology for compressing refrigerants and in conditioning.

The design scheme of the rotary vane compressor is shown in Figure 1. The working cell is limited by two successive vanes, the outer surface of the rotor, the inner surface of the cylinder, and end walls. During the rotation of the eccentric mounted rotor, the vanes, which are freely located inside the grooves, are thrown by the centrifugal forces to the periphery, thus dividing the free sickle-shaped cavity into separate working cells. In the left half-plane, an increase in the area (and, respectively, volume) of the cell occurs, in the right half, there occurs a decrease in the cell.

\footnotetext{
${ }^{*}$ Corresponding author: kotlov_andrej@mail.ru
} 


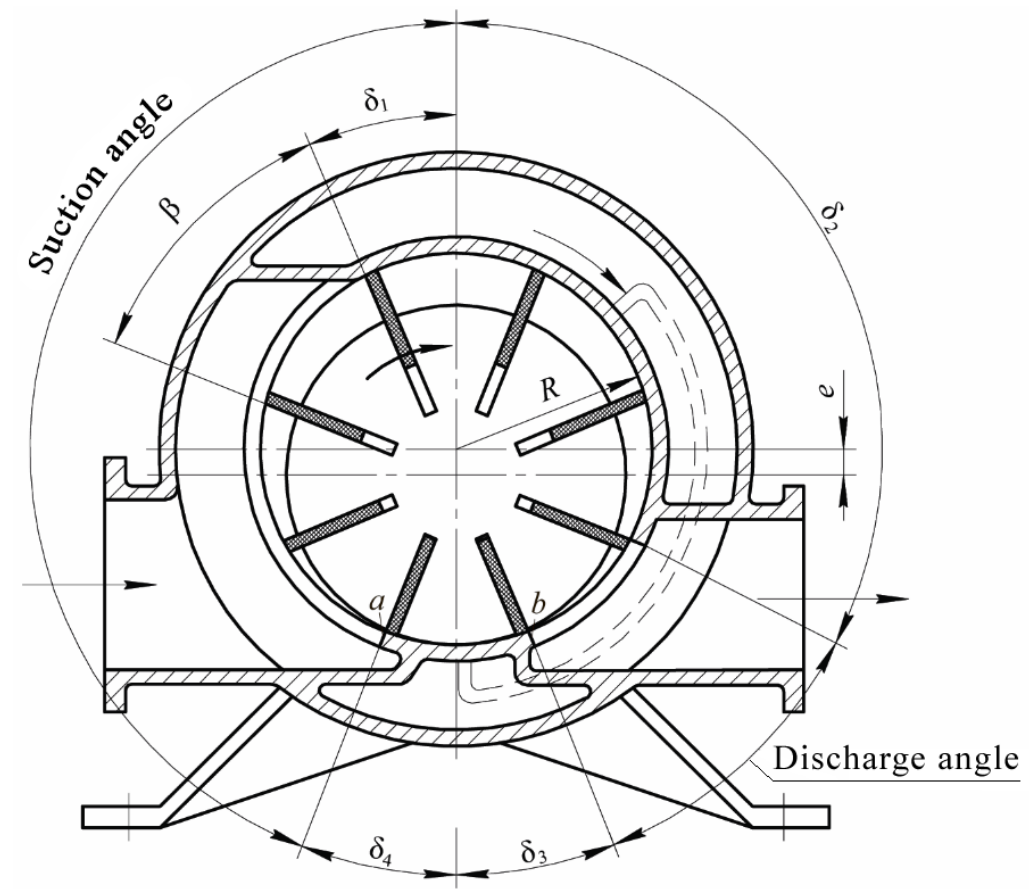

Fig. 1. The design scheme of the rotary vane compressor

At the intersection of the edge " $a$ " by the front vane of the working cell, fresh gas begins to flow into the cell. Suction finishes when the end vane of the cell crosses the upper edge of the suction window. With further rotation of the rotor in the working cell, compression occurs due to the decrease of the cell volume. Upon reaching the upper edge of the discharge window by the front vane, the cell is connected to the discharge window and gas is injected. The discharge continues until the end vane closes the discharge window when crossing the " $b$ " edge. Gas that is not displaced from the cell during the discharge time is clamped in the pass volume to the lowest point of the cylinder. Then the volume of the cell begins to increase, and a vacuum appears in it.

Rotational compressors are constantly improved, their volumetric and energy characteristics, reliability and durability, degree of automation are increased, at the same time dimensions and metal consumption, noise and vibration are reduced. When designing rotary machines, various tasks arise in the search for more efficient and reliable design solutions related to: the organization of the working process $[8,9]$; the choice of constructive forms of the flow part [10]; calculation of the strength of machine elements and the choice of material [11]. The range of compressors usage according to their performance and final pressure is expanding, designs and manufacturing technology are being improved.

The purpose of the work is to increase the efficiency of the working process of a rotary vane compressor by optimizing the number of vanes.

\section{Research method}

Within the framework of the study, a mathematical model and a computer program were developed, which made it possible to calculate the preliminary dimensions of the RVC based on the available initial data. The RVC performance, initial and final pressure, initial temperature, thermodynamic parameters of gas were considered as initial data. 
The actual mass $\dot{m}_{a}$ productivity of the compressor is less than theoretical one $\dot{m}_{t}$, i.e. $\dot{m}_{a}=\lambda \dot{m}_{t}$ where $\lambda<1$ is the performance factor $[12,13]$.

The performance factor for the RVC can be represented as (1)

$$
\lambda=\lambda_{p} \cdot \lambda_{t} \cdot\left(1-v_{u}\right)-v_{l}-v_{e l},
$$

where $\lambda_{p}$ is the pressure factor, $\lambda_{t}$ is the temperature factor, $v_{u}$ is the operation factor, $v_{l}$ is the leakage factor, $v_{e l}$ is the external leakage factor.

The pressure factor is a coefficient that takes into account pressure losses during the passage of gas through the suction window. The factor $\lambda_{t}$ takes into account the rise in temperature of the suction gas. An increase in temperature leads to a decrease in the density of the suction gas and, consequently, to a decrease in productivity. The coefficient $v_{u}$ shows how much of the theoretically possible volume is used in the suction process. The coefficient $v_{1}$ takes into account the leakage of gas from the cavities with high pressure to the cavities with low pressure. Leakage reduces the volume of suction gas, as they themselves occupy a part of the volume of the suction cavity and heat up the suction gas. The coefficient $v_{\mathrm{el}}$ takes into account the volume of gas that flows from the working cavity of the compressor in the ambience.

Preliminary, the components of the performance factor are selected on the basis of available recommendations. A preliminary thermodynamic calculation is made and the initial geometrical parameters are determined. Using these values and the formula (2), the optimal number of vanes is calculated, based only on considerations of achieving the maximum theoretical performance [14].

$$
z_{\mathrm{opt}}=\pi \sqrt[3]{\frac{R\left(2 \frac{e}{R}+1\right)}{3 b}}
$$

where $R$ is the stator radius (see Figure 1), $e$ is eccentricity, $b$ is the vane width.

In fact, the number of vanes ambiguously affects the integral characteristics of the compressor. On one hand, the mass transfer between the cavities decreases, which leads to a decrease in relative overflows, the pressure drop on the vane decreases (the load on the vane decreases and the friction loss decreases), on the other hand, the overall friction power increases and the mechanical and indicator efficiencies decrease.

We consider the influence of the number of vanes on the mass transfer between the working cells and the suction cavity. The calculation of the mass flow rate was carried out according to the formula of S.E. Zakharenko (3) [15-18], which takes into account the shape of the gap; its geometric dimensions; flow parameters before and after the gap; gas friction in the gap; loss of pressure at the inlet and outlet of the gap. For example, figure 2 shows a rectangular gap and its main dimensions; gap width (gap) $\delta$ is the smallest gap size in a plane perpendicular to the flow; the gap length $l$ is the size in the plane A; the gas path length $b$ is the size of the gap in the direction of gas motion (in the B plane).

$$
\dot{m}=\mu \delta l \sqrt{\frac{\rho_{1}^{*} p_{1}\left(\varepsilon^{2}-1\right)}{\ln \varepsilon^{2}+\xi+\lambda_{r} \Sigma}},
$$

where $\mu$ is the discharge coefficient (we took $\mu=0.8$ ); $\delta$ is clearance in the gap; $l$ is the gap depth; $\rho_{1}^{*}=p_{1} / R T_{2}$ is conventional density; $\mathrm{p}_{1}$ is the gas pressure behind the gap; $T_{2}$ is the gas temperature in front of the gap; $\varepsilon$ is the pressure ratio in front of the gap and behind it, $p_{2} / p_{1}$; $\xi$ is the total coefficient of local resistances at the entrance and exit of the gap (for gaps with a smooth variation of the cross section $\xi=1.418$, for gaps with a constant width, instantaneous narrowing at the input and instantaneous expansion at the exit $\xi=2.5$ ); $\lambda \mathrm{r}$ is the friction factor [19], $\Sigma$ is the form factor. 


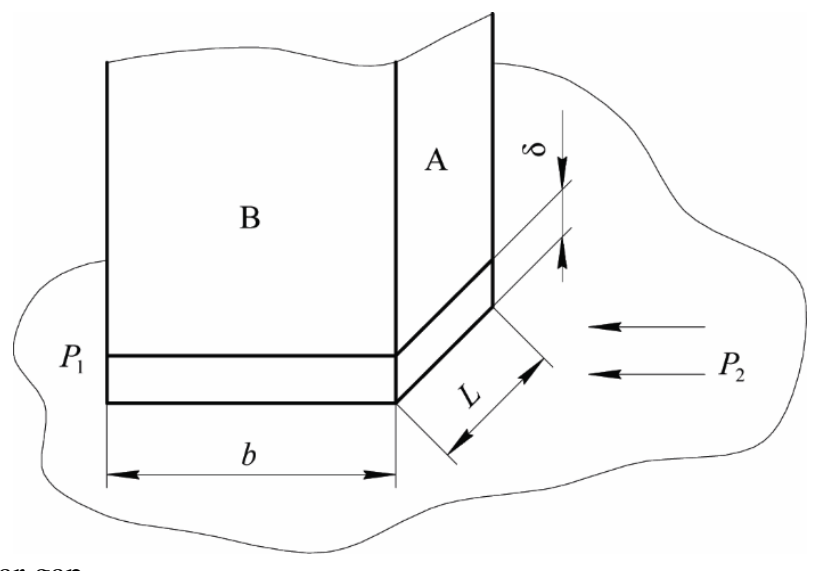

Fig. 2. A rectangular gap

Equation (3) cannot be solved, since the friction factor $\lambda_{r}$ entering into the equation depends on the mass flow rate. The solution is sought by the method of successive approximations. For the initial approximation one takes the flow rate at the critical discharge, which is determined by the formula of Saint-Venant-Wenzel (4)

$$
\dot{m}_{0}=\mu \delta l p_{2}\left(\frac{2}{k+1}\right)^{\frac{1}{k-1}} \sqrt{\frac{2 k}{k+1} \frac{1}{R T_{2}}},
$$

where $k$ is the adiabatic factor, $R$ is the gas constant $T_{2}$ is the temperature in front of the gap.

The $\operatorname{Re}_{0}$ number is determined from the obtained value of the flow rate using the formula (5) [20]

$$
R e_{0}=\frac{2 \dot{m}_{0}}{\mu_{v}(\delta+l)}
$$

where $\mu_{v}$ is the dynamic viscosity of gas.

Depending on the Re number, the friction factor is found and the obtained value is substituted into (3). In this way, the first approximation of mass flow rate $\dot{m}_{1}$ is found. Further it is substituted into the formula for calculating Re, friction factor is found again, etc. until condition (6) is fulfilled.

$$
\frac{\left|\dot{m}_{i+1}-\dot{m}_{i}\right|}{\dot{m}_{i}} \geq \Delta
$$

where $\Delta$ is the preset accuracy of calculation.

In order to compare not only qualitative but also quantitative results of the calculation, we use a compressor with a capacity of $10 \mathrm{~m}^{3} / \mathrm{min}$ as a test object, which compresses gas with an initial pressure equal to the atmospheric pressure, up to an absolute final pressure of $0.4 \mathrm{MPa}$.

Internal gas overflows in a rotary compressor occur mainly through radial clearances (see Figure 3a) and end clearances (see Figure 3b) and from gas transfer in an isolated volume (see Figure 3c). 


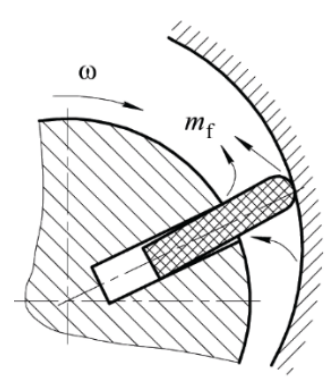

a

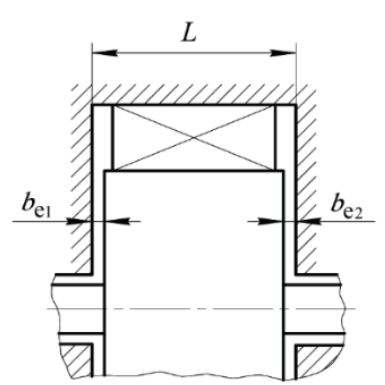

b

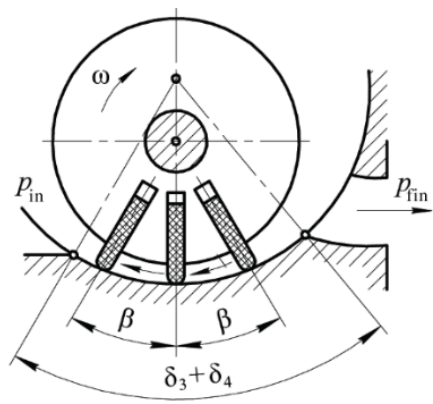

c

Fig. 3. Gas overflow: $a$-radial overflows and overflows through gaps; $b$-end overflows through the clearances $b_{\mathrm{e} 1 \text { и }} b_{\mathrm{e} 2} ; c$-overflows from isolated volume

The specified overflow performance coefficient through the radial gaps in the upper part of the cylinder (see Figure 1) is affected only by the leakage through the vane located on the back side of the cell disconnected from the suction chamber. The direction of overflows through this vane depends on the location of the upper edge of the suction window. If the cell is cut off from the suction window until it reaches its maximum position, then the overflows will go from the suction chamber to the cell until the volume of this cell becomes less than the maximum possible. With further rotation of the rotor, the direction of flow of the overflows will change to the opposite. Let the upper edge of the suction window be fixed and shifted down. The angle of deviation of the bisector from the vertical of the initial cell $\delta_{1}$ is chosen to be $30^{\circ}$. It is assumed that the pressure in this cell is equal to the suction pressure until the bisector of the cell reaches the zero position. Mass transfer occurs at the angle of rotation equal to $\beta$ ( $\beta=2 \pi / \mathrm{z}$ is the angle of cell opening). For the investigated version of the compressor, the overflow from the initial cell is insignificant and does not exceed $1 \%$. As the number of vanes increases, the mass transfer time decreases, and the total mass flow decreases accordingly. Since the overflows from the first cell to the previous one through the radial gap is extremely small, they can be neglected.

In addition, there are overflows through the radial and end clearances from the cell located directly in front of the suction chamber. The pressure in this cell for the angle of rotation 180 $\leq \varphi \leq 180+\beta$, is determined by the formula (7).

$$
p_{\varphi}=p_{\text {fin }}\left(\frac{\beta+2 \sin \frac{\beta}{2}+\frac{\mathrm{e}}{2 \mathrm{R}} \sin \beta-\frac{\mathrm{e}}{2 \mathrm{R}} \beta}{\beta+2 \cos \varphi \sin \frac{\beta}{2}+\frac{\mathrm{e}}{2 \mathrm{R}} \cos 2 \varphi \sin \beta-\frac{\mathrm{e}}{2 \mathrm{R}} \beta}\right)^{\mathrm{n}},
$$

where $p_{\text {fin }}$ is the final pressure.

The lowest edge of the suction window is also fixed. The displacement of the bisector of the last cell from the lower point of the stator is assumed to be $30^{\circ}$ in the direction of rotation of the rotor. Overflows increase with decreasing the number of vanes. However, due to the higher pressure in the lower cells and a shorter gap depth, this mass transfer is more significant (see Figure 4). 


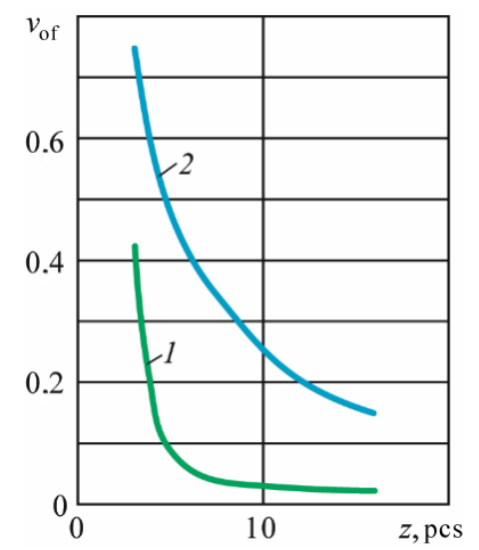

Fig. 4. End overflows from the first (1) and the last (2) cells to the suction cavity

At the lower part of the stator, with a small number of vanes, gas can be transferred with discharge parameters from the clamped volume to the suction chamber (see Figure $3 \mathrm{c}$ ). This mass flow, if $\left(\delta_{3}+\delta_{4}\right)>2 \beta$, is calculated by the formula (8).

$$
\dot{m}_{\text {is }}=F_{\text {is }} z \operatorname{Ln} \rho_{\text {dis }} \text {, }
$$

where $F_{\text {is }}$ is the end area of the isolated cell, $n$ is the rotor frequency; $\rho_{\text {dis }}$ is the density of the discharged gas.

If $\varphi=180-\delta_{3}+\beta / 2$, the end area of the isolated cell is determined according to the formula (9), in other cases $\dot{m}_{\text {is }}=0$.

$$
F_{\text {is }}=\mathrm{eR}\left(\beta+2 \cos \varphi \sin \frac{\beta}{2}+\frac{\mathrm{e}}{2 \mathrm{R}} \cos 2 \varphi \sin \beta-\frac{\mathrm{e}}{2 \mathrm{R}} \beta\right)
$$

Overflows from an isolated volume strongly depend on the position of the lower edge of the discharge window. The lower is this edge, the smaller the pinched volume is, and the smaller the number of overflows in the suction chamber is. With a fixed lower edge, an increase in the number of vanes leads to significant losses: thus, the displacement of the lower edge of the discharge window by $\delta_{3}=60^{\circ}$ leads to an increase in mass leakages up to $30 \%$ for a large number of vanes.

Overflow through the grooves is extremely small due to the large hydraulic resistance. The main overflows occur through the end gaps $\left(\delta_{\mathrm{e} 1}, \delta_{\mathrm{e} 2}\right)$ between the rotor and the caps (see Figure $3 b$ ).

The process of mass transfer that occurs through the end surface of the rotor should be divided into two stages: flow at the stage of compression in the working cells with variable parameters at the inlet to the gap; overflow at the stage of discharge with gas parameters (pressure, temperature) before the gap, equal to these parameters in the discharge pipe. To do this, one first need to determine the angle of compression $\varphi_{\text {comp }}$, i.e. the moment of the beginning of the discharge. The condition for finding this moment is the achievement of discharge pressure in the working cell, the corresponding angle is determined by the formula (10)

$$
\varphi_{\text {comp }}=\arccos \left[\frac{2}{\left(\frac{p_{\text {fin }}}{p_{\text {in }}}\right)^{\frac{1}{n}}}-1\right],
$$

where $n$ is the polytropic index.

The gap parameters (see Figure 2) for the overflow calculation through the end clearances are determined according to the formulae (11) and (12).

$$
l=2 r \sin \varphi \sin \frac{\beta}{2} \text {. }
$$




$$
b=2 r \sin \varphi \cos \frac{\beta}{2}
$$

\section{Results and discussion}

According to formula (10), the compression angle depends only on the ratio between the final and initial pressure. Therefore, the number of vanes practically does not affect its variation. The mass flow rate of gas overflows in compression along the rotation angle of the rotor (see Figure 5) increases noticeably with a decrease in the number of vanes. There is a clear trend of increasing the mass transfer when the cell approaches the opening of the discharge window, and for a smaller number $\mathrm{z}$ this tendency is more obvious.

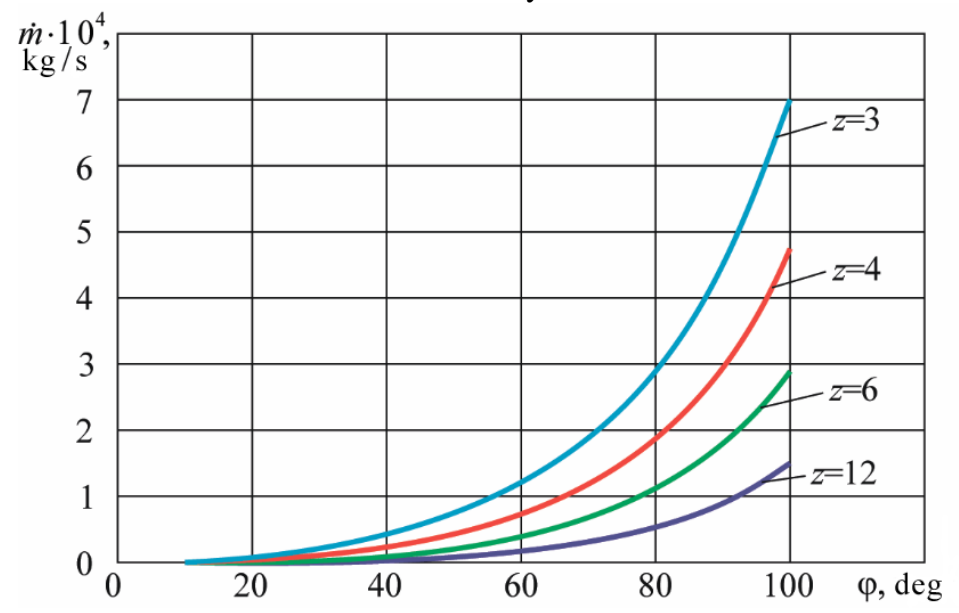

Fig. 5. End overflows at compression

The minimum volume of the working cell is determined by the angle $\beta / 2$. When designing, it is recommended to choose the position of the lower edge of the discharge window in the range from $\beta / 2$ to $\beta$. The question of pumping overflows arises due to the accepted assumptions. The edge will always depend on $\beta / 2$, and an increase in the number of vanes will lead to a lower bottom edge, and, consequently, to an increase in the duration of the mass transfer process and the amount of gas flowing. The results are shown in Figure 6, from which it is seen that an increase in the number of vanes leads to an increase in overflows.

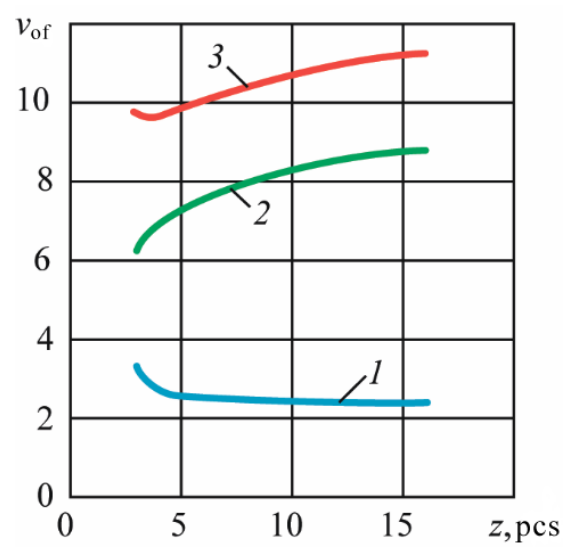

Fig. 6. Mass transfer at alternating bottom edge of the discharge window: 1 - during compression; $2-$ during discharge; 3 - total 
Further it was assumed that the lower edge is fixed as well as the upper one. Otherwise, with a small number of vanes and with a constant upper edge of the discharge window, it will actually be absent. It was assumed that the angle $\delta_{3}$ for all variants is constant and equal to $60^{\circ}$. In this case, an increase in the number of vanes leads to a decrease in mass transfer during discharge and total mass transfer (see Figure 7).

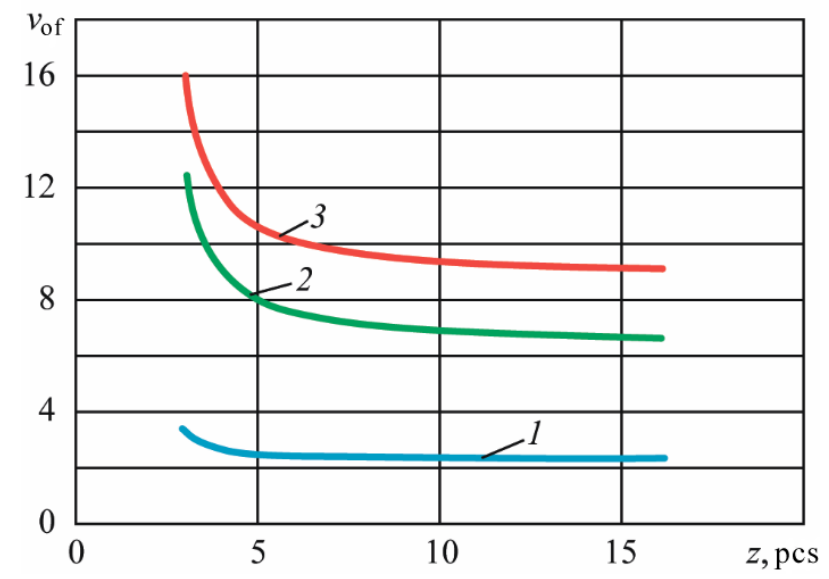

Fig. 7. Relative overflows with a constant lower edge of the discharge window: 1 - during compression; 2 - during discharge; 3 - total

A further increase in the number of vanes does not lead to a noticeable change in the relative overflow. In quantitative terms, end overflows prevail over all others (see Figure 8).

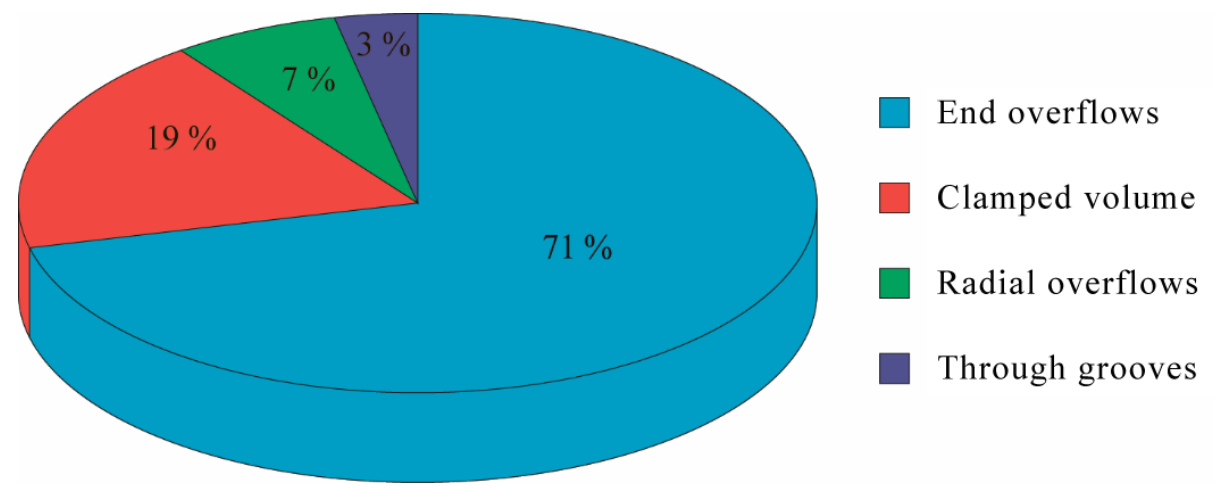

Fig. 8. The fractions of relative overflows in the total mass transfer

\section{Conclusions}

When choosing the location of the lower edge of the discharge window, it is necessary to solve an optimization problem. On one hand, lowering the lower edge leads to a decrease in overflows from the pinched volume at $\mathrm{z}>10$, and on the other hand, this increases the discharge process time, which leads to an increase in end overflows. To control mass transfer from the isolated volume a gas bypass from the discharge to the suction is created. At such design the pressure in the bulk volume is slightly higher than the suction pressure, and the influence of the clamped volume and overflow from the last cell can be neglected.

Thus, using the developed methodology for determining the performance of RVC, taking into account the end overflows, inter-chamber overflows and mass transfer in a pinched volume, it is possible to choose the optimal number of vanes in order to increase compressor 
performance. It is established that the most important are the overflows through the end gaps between the rotor and the stator.

The developed mathematical model and computer program allows one to carry out both preliminary design of RVC and verification calculations with the search for optimal values of the number of vanes.

The research was performed by a grant of Russian Science Foundation (project No. 18-79-10165).

\section{References}

1. A. Shouman, A. Hussin, A. Hamed, N. Mahmoud, A.E. Baz, IOP Conference Series: Materials Science and Engineering, 232(1), 012060 (2017)

2. G. Bianchi, R. Cipollone, Applied Energy, 142, 95-107 (2015)

3. R. Cipollone, G. Valenti, G. Bianchi, G. Contaldi, T. Calvi, Proceedings of the Institution of Mechanical Engineers, 229 (2), 96-103 (2015)

4. R. Cipollone, G. Valenti, G. Bianchi, G. Contaldi, T. Calvi, 8th International Conference on Compressors and Their Systems, 173-181 (City University, London, 2013)

5. R. Cipollone, G. Bianchi, G. Contaldi, ASME International Mechanical Engineering Congress and Exposition, Proceedings, 6, 69-80 (2012)

6. E.A Pavlyuchenko, V.E Shcherba, A.P. Bolshtyanskij, E.A. Lysenko, A.K. Kuzhbanov, Omskij nauchnyj vestnik mashinostroenie i mashinovedenie, 2 (100), 118-121 (2011)

7. G. Bianchi, S. Rane, A. Kovacevic, R. Cipollone, Advances in Engineering Software, 112, 180-191 (2017)

8. I.G. Hisameev, V.A. Maximov, Double rotor screw and spur compressors. Theory, calculation and design (2000)

9. A. Kovacevic, M. Arjeneh, S. Rane, N. Stosic, M. Gavaises, International conference on Screw Machines, 309-318 (Dortmund, Germany, 2014)

10. S. Herlemann, J. Hauser, N. Henning International conference on Screw Machines, 211-224 (Dortmund, Germany, 2014)

11. R. Schuhmann, H. Ochs, R. Missal, T. Schmitt, International Conference on Screw Machines, 15-28 (Dortmund, Germany, 2014)

12. M. Grieb, A. Brümmer, International Conference on Screw Machines, 197-210 (Dortmund, Germany, 2014)

13. I.A. Sakun, Screw Compressors (1970)

14. E.S. Frolov, I.V. Avtonomova, V.I. Vasiliev, Mechanical Vacuum Pumps (1989)

15. S.E. Zakharenko, Trudy of the Leningrad Polytechnic Institute named after M.I. Kalinin, 249, 69-75 (1965)

16. B. S. Hrustalev, Dis... d-ra. tekhn. nauk 377, (1999)

17. A.A. Kotlov, Dis... kand. tekhn. nauk 138, (2011)

18. V.N. Dokukin, V.A. Pronin, Nauchnyj zhurnal NIU ITMO. Seriya «Holodil'naya tekhnika i kondicionirovanie», 3, 17-23 (2014)

19. M.Yu. Elagin, E.M. Sidorov, Izvestiya TulGU. Tekhnicheskie nauki, 6, 88-96 (2014)

20. V.A. Kosachevskij, Vestnik MAH, 4, 40-46 (2016) 